\title{
Estudio de la Formacion de los Complejos Intermedios Durante la
}

\section{Sintesis de Alumina}

\author{
J.E. Rodríguez-Páez ${ }^{\mathrm{a}}{ }^{*}$, C. Villaquirán ${ }^{\mathrm{a}}$, J. Cobo $^{\mathrm{b}}$ \\ ${ }^{a}$ Departamento de Física, Universidad del Cauca, Popayán - Cauca, Colombia \\ ${ }^{\mathrm{b}}$ Departamento de Química, Universidad del Cauca, Popayán - Cauca, Colombia
}

Received: January 11, 2001; Revised: September 22, 2001

\begin{abstract}
One of the most important compounds that are used in structural ceramics is alumina. During the last years, a variety of synthesis methods have been developed to obtain raw materials with suitable characteristics in terms of particle composition, size and shape. Controlled precipitation method (CP) allows to reach these demands and with this method is possible to control the synthesis to guarantee characteristics of the ceramic powder. One stage of the CPM is the formation of intermediate complexes of cations whose oxide derives to obtain. In this work the results of study of the formation of aluminum intermediate complexes are presented. We used potentiometric and conductimetric titration to follow the advance of the process. We utilized UV-visible and IR spectroscopies to characterize the liquid phase present in the system.
\end{abstract}

Keywords: synthesis, controlled precipitation, alumina, intermediate complexes

\section{Introducción}

En la tecnología actual la alúmina, $\mathrm{Al}_{2} \mathrm{O}_{3}$, es uno de los compuestos más utilizados ${ }^{1}$. Tiene múltiples usos en la industria; como aditivo en la industria del papel, dentífricos, pinturas, recubrimientos, en la industria del caucho y de polímeros. La alúmina activada se utiliza como desecante y en procesos catalíticos. En la industria electrónica se emplea en equipos para acumulación y generación de potencia eléctrica y en la fabricación de porcelana aislante. Se utiliza además, en la elaboración de recubrimientos y en la fabricación de biomateriales.

La bauxita es la más importante y abundante materia prima para los polvos de alúmina y el proceso Bayer el que se utiliza con tal fin ${ }^{2}$. El producto que se obtiene contiene impurezas indeseables de $\mathrm{Na}_{2} \mathrm{O}, \mathrm{SiO}_{2}, \mathrm{Fe}_{2} \mathrm{O}_{3}$, etc., no adecuadas para los requerimientos tecnológicos actuales. Con el fin de obtener una alúmina más pura, y con un tamaño de partícula y morfología determinados, se han desarrollado una gran variedad de métodos ${ }^{3}$, destacándose entre ellos la precipitación a partir de soluciones homogéneas $^{4,5}$, el de emulsiones ${ }^{6}$ y sol-emulsión-gel ${ }^{7}$. Los precursores del catión aluminio más utilizados son el sulfato, $\mathrm{Al}_{2}\left(\mathrm{SO}_{4}\right)_{3}$, los alcóxidos, $\mathrm{Al}\left(\mathrm{OC}_{3} \mathrm{H}_{7}\right)_{3}, \mathrm{Al}\left(\mathrm{OC}_{4} \mathrm{H}_{9}{ }^{\mathrm{s}}\right)_{3} \mathrm{o}$ $\mathrm{Al}\left(\mathrm{OC}_{5} \mathrm{H}_{11}{ }^{\mathrm{t}}\right)_{3}$ y el cloruro de aluminio $\mathrm{AlCl}_{3}$. Aunque las técnicas que permiten obtener la alúmina $\mathrm{Al}_{2} \mathrm{O}_{3}$, con dife-

*e-mail: jnpaez@ucauca.edu.co rente morfología y tamaño de partícula ${ }^{8-11}$, son descritas adecuadamente por sus autores es necesario conocer más sobre los principios físico-químicos involucrados en ellas $^{12}$. Debido a que los métodos de síntesis que permiten la formación de una fase sólida en el seno de una solución acuosa son los de mayor posibilidad industrial ${ }^{13}$, y que el procedimiento utilizado en este trabajo utiliza esta metodología, es necesario conocer más sobre la formación de las especies polinucleares y poliméricas del aluminio, su naturaleza, y su participación en la conformación de los núcleos de fase sólida.

La formación de las partículas coloidales de compuestos de aluminio, dopois de $\mathrm{Al}^{3+}$, oxihidroxidos de aluminio por ejemplo, en soluciones acuosas de $\mathrm{Al}^{3+}$ involucra una serie de pasos durante los cuales se forman complejos cuyo tamaño y complejidad estructural se incrementa a medida que avanza el proceso de adición del reactivo que favorece la hidrólisis de la solución ${ }^{14}$. La adición del compuesto, una base inorgánica u orgánica, hidroliza la solución y ocasiona la precipitación de una sal básica cuya estructura es de especial interés para obtener información sobre los compuestos polinucleares que existen en las soluciones de una sal de aluminio hidrolizada ${ }^{15}$. Bottero y Fiessinger ${ }^{16}$ trabajando con soluciones de cloruro de aluminio, y utilizando dispersión de rayos-x a pequeño ángulo, resonancia magnética nuclear y valoración poten- 
ciométrica para estudiar los productos de hidrólisis que se forman al adicionar una base, determinaron la presencia de especies hidratadas de aluminio tetra-coordinado, $\mathrm{Al}(\mathrm{OH})_{4}{ }^{-}$, de especies mononucleares $\left(\mathrm{Al}\left(\mathrm{H}_{2} \mathrm{O}\right)_{6}{ }^{3+}\right)$, $\mathrm{Al}\left(\mathrm{H}_{2} \mathrm{O}\right)_{5} \mathrm{OH}^{2+}$ y $\left.\mathrm{Al}\left(\mathrm{H}_{2} \mathrm{O}\right)_{4}(\mathrm{OH})_{2}^{+}\right)$, y del policatión $\mathrm{Al}_{12}{ }^{\mathrm{VI}}(\mathrm{OH})_{24} \mathrm{Al}^{\mathrm{IV}} \mathrm{O}_{4}\left(\mathrm{H}_{2} \mathrm{O}\right)_{12}{ }^{7+}$, denominado ion $\mathrm{Al}_{13}{ }^{7+}$, identificado y descrito por Johansson ${ }^{15}$. La presencia de una especie u otra en el sistema, está determinada por la razón base adicionada: concentración del precursor, o sea por el pH de la solución ${ }^{16}$. La concentración del ion $\mathrm{Al}_{13}{ }^{7+}$ se incrementa hasta alcanzar un máximo entre $\mathrm{pH} 4.06 \mathrm{y}$ $4.28^{14,16}$. Su posterior hidrólisis ocasiona la condensación del policatión y la formación de grandes agregados.

Si la solución de cloruro de aluminio es hidrolizada con hidróxido de amonio y sulfato de amonio se obtienen cristales tetraédricos de $\left(\mathrm{NH}_{4}\right)_{7}\left[\mathrm{Al}_{13} \mathrm{O}_{4}(\mathrm{OH})_{24}\left(\mathrm{H}_{2} \mathrm{O}\right)_{12}\right]\left(\mathrm{SO}_{4}\right) \bullet \mathrm{xH}_{2} \mathrm{O}{ }^{15,17}$. Por otro lado, cristales tipo plaqueta monoclínicos de sulfato básico de aluminio fueron obtenidos hidrolizando una solución de cloruro de aluminio con sulfato de sodio ${ }^{15}$, la composición del sulfato básico fue $13 \mathrm{Al}_{2} \mathrm{O}_{3} \bullet 6 \mathrm{SO}_{3} \bullet \mathrm{xH}_{2} \mathrm{O}$.

La auto-hidrólisis de soluciones de sulfato de aluminio ha permitido obtener partículas esféricas monotamaño cuando se ejerce un fuerte control sobre la concentración del precursor, naturaleza de los aditivos y temperatura $^{4,5,9,10,11}$. La lenta adición gota a gota, de $\mathrm{NH}_{4} \mathrm{OH}$ por ejemplo, a la solución de la sal de aluminio en continua agitación, también permite la obtención de partículas esféricas ${ }^{18}$. Si la adición de la base es rápida, las partículas que precipitan tienen una forma de aguja y si las soluciones de sulfato saturadas son evaporadas o enfriadas, las partículas adquieren una forma de cubos o plaquetas ${ }^{19}$.

Cambiando uniformemente el $\mathrm{pH}$ de la disolución, la reacción se produce más rápido y permite una mayor producción de esferas. Para lograr el cambio lento y uniforme del $\mathrm{pH}$ se utiliza úrea o formamida, las cuales se disuelven en soluciones que posteriormente se calientan ${ }^{4,5,9,10,11,18}$. El precipitado que se forma, durante el período de envejecimiento del sistema, es un sulfato básico hidratado de aluminio $^{4,9,10}$. No se conoce ningún reporte que indique la obtención directa de óxido de aluminio a partir de soluciones acuosas. En principio, soles de oxihidróxido de aluminio con morfología esférica, se pueden obtener si las soluciones de la sal de aluminio contiene ciertos aniones, iones de sulfato por ejemplo, y son envejecidas a elevadas temperaturas ${ }^{5,11,20}$. Hay que controlar, sin embargo, la concentración de la sal de aluminio (precursor), la concentración de sulfato, el pH inicial, la temperatura final y el calentamiento de la solución, para que la autohidrólisis del aluminio combinado con el acomplejamiento del sulfato permita obtener partículas esféricas con relativa alta uniformidad $^{20}$.
Aunque el calentamiento de soluciones de sulfato de aluminio, y su posterior envejecimiento, permite obtener soles de oxihidróxido con una relativa distribución estrecha de tamaño ${ }^{20}$, el diámetro de las partículas obtenidas para la misma concentración puede variar considerablemente. Esto se puede justificar considerando que la nucleación de una fase sólida es muy sensible a los pequeños cambios en las condiciones experimentales $4,9,10,11,20$.

$\mathrm{El}$ ion aluminio se hidroliza y los iones sulfato tienden a coordinarse con los iones hidrolizados de aluminio para formar especies complejas ${ }^{21}$. Aunque el ion sulfato en otros sistemas, soles de oxihidróxido de cromo por ejemplo ${ }^{22}$, actúa como puente entre especies polinucleares hidrolizadas formando enlaces muy fuertes, en los oxihidróxidos de aluminio no cumple esta labor, lo que permite que los iones sean fácilmente intercambiables ${ }^{20}$. Willard ${ }^{18}$ encontró que los precipitados de sulfato básico de aluminio pierden prácticamente todo el sulfato por digestión del sólido obtenido a $\mathrm{pH}$ 9.4. Bajo ciertas condiciones, los sulfatos básicos de aluminio son menos solubles que el hidróxido de aluminio o el sulfato de aluminio, por lo que deben ser estos complejos básicos los que actúen como precursores en la formación de los embriones y posteriormente de los núcleos de la fase sólida ${ }^{20}$. Esto indica que uno o más de los complejos de sulfato básico de aluminio pueden actuar como precursores de la fase sólida; entre ellos se destaca el de composición $\mathrm{Al}_{4}(\mathrm{OH})_{10}\left(\mathrm{SO}_{4}\right)$ que ha sido sugerido reiteradamente por varios autores ${ }^{23}$.

En este trabajo se presentan los resultados obtenidos al analizar una de las etapas del proceso de precipitación controlad (MPC) que se utiliza en la síntesis de $\mathrm{Al}_{2} \mathrm{O}_{3}$. El estudio se centró en la formación de complejos y compuestos de alúmina, en el sistema $\mathrm{Al}_{2}\left(\mathrm{SO}_{4}\right)-\mathrm{NH}_{4} \mathrm{OH}-\mathrm{H}_{2} \mathrm{O}$. La evolución del proceso se registró en curvas de valoración potenciométrica y conductimétrica. La fase líquida presente en el sistema, a diferentes $\mathrm{pH}$, se caracterizó utilizando UV-visible y espectroscopia infrarroja (FTIR) y para caracterizar la fase sólida, presente en el sistema, se utilizó espectroscopia infrarroja y difracción de rayos-X (DRX)

\section{Procedimiento Experimental}

\subsection{Valoración potenciométrica y conductimétrica}

Se realizó la valoración potenciométrica, a $25^{\circ} \mathrm{C}$, de soluciones acuosas con diferente concentración de sulfato de aluminio, $\mathrm{Al}_{2}\left(\mathrm{SO}_{4}\right)_{3} \bullet 18 \mathrm{H}_{2} \mathrm{O}$ (Carlo Erbo), $0.02 \mathrm{M}$, $0.1 \mathrm{M}$ y $0.3 \mathrm{M}$, utilizando $\mathrm{NH}_{4} \mathrm{OH}$ (marca Merck) $15 \mathrm{M}$. Para conocer los valores de $\mathrm{pH}$ del sistema se utilizó un pH-metro Metrohm 744 con electrodo de vidrio Ref. 6.0259.100 (calibrado con soluciones tampón a pH 7 y 4). El sistema se conformó disolviendo el $\mathrm{Al}_{2}\left(\mathrm{SO}_{4}\right)_{3}$ en 
$200 \mathrm{~mL}$ de agua destilada-desionizada. La disolución se mantuvo en continua agitación a 400 r.p.m. y al tornarse completamente transparente se comenzó a adicionar lentamente $\mathrm{NH}_{4} \mathrm{OH}$, utilizando para ello un Dosimat E-775 marca Metrohm. Se llevó el sistema hasta valores de $\mathrm{pH}$ altos donde no se presentó una variación apreciable de este parámetro.

Simultáneamente a la valoración potenciométrica se realizó la valoración conductimétrica. Para ello se utilizó un conductímetro marca Orion 410A. Los dos electrodos, el del pH-metro y el del conductímetro, se colocaron en el interior del sistema y se registraron los cambios de $\mathrm{pH}$ y de conductividad como funciones del volumen adicionado de $\mathrm{NH}_{4} \mathrm{OH}$ : el sistema se llevó hasta un valor de $\mathrm{pH}$ de aproximadamente 10. La Fig. 1a muestra los resultados de valoración potenciométrica y conducimétrica para una disolución acuosa de $\mathrm{Al}_{2}\left(\mathrm{SO}_{4}\right)_{3}$ de $0.02 \mathrm{M}$.

\subsection{Caracterización del sistema}

Para determinar las fases cristalinas en el sólido y los grupos funcionales presentes tanto en el líquido como en el sólido, a un cierto valor de $\mathrm{pH}$, se tomó la suspensión coloidal obtenida y se filtró al vacío separando la fase líquida de la fase sólida. Al líquido se le realizó espectroscopía UV-visible e infrarrojo (FTIR) y el sólido se caracterizó utilizando FTIR y DRX.

\section{Resultados y Discusión}

\subsection{Valoración potenciométrica}

La curva de valoración potenciométrica de la Fig. 1a muestra tres regiones muy bien definidas: dos pasos ( $\mathrm{AB}$ y $\mathrm{CD})$ y un salto $(\mathrm{BC})$. La primera región $\mathrm{AB}$ corresponde a una zona prácticamente plana, con poca variación de $\mathrm{pH}$ y alto consumo de $\mathrm{OH}^{-}$por parte del sistema. La segunda, $\mathrm{BC}$, muestra una marcada variación de $\mathrm{pH}$ y la última, $\mathrm{CD}$,

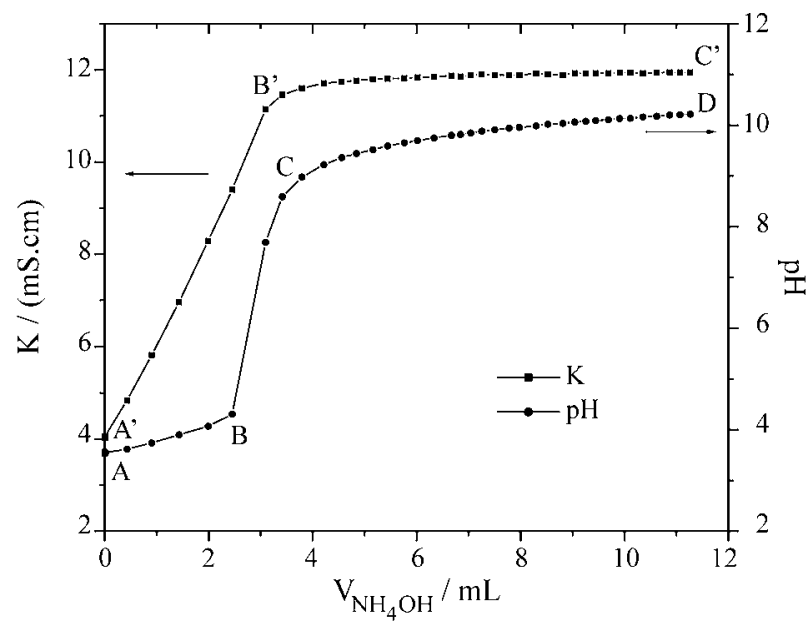

Figure 1a. Curvas potenciométrica y conductimétrica para una disolución 0.02 molar de $\mathrm{Al}_{2}\left(\mathrm{SO}_{4}\right)_{3}$. corresponde a la región de saturación y ocurre a un valor de $\mathrm{pH}$ aproximadamente 10 .

Desde el punto A hasta el punto B el sistema se torna opaco debido a la presencia de pequeños "copos de nieve", con forma no muy bien definida, los cuales se mantienen durante la adición de $\mathrm{NH}_{4} \mathrm{OH}$. Considerando el esquema de Tezak ${ }^{24}$, y debido a la alta cantidad de iones $\mathrm{OH}^{-}$que se enlazan a los iones aluminios en esta región, se puede considerar que a través de las reacciones químicas entre las especies fundamentales, $\mathrm{Al}^{3+}, \mathrm{SO}_{4}{ }^{2-}, \mathrm{OH}^{-}$y $\mathrm{NH}_{4}{ }^{+}$, hidratadas se generarían las especies mono, polinucleares y poliméricas que estructuran los embriones de la fase sólida. Posteriormente se conformarían los núcleos, tal como se indica en la literatura ${ }^{25}$. De acuerdo a lo mencionado, en esta primera región el proceso que predomina es el de nucleación y por lo tanto la formación de la fase sólida en el seno del sistema.

El salto BC no es muy abrupto y es por esto que no se puede determinar exactamente el punto de equivalencia correspondiente de la curva de valoración potenciométrica en función del volumen adicionado, Fig. 1b. A partir de un valor de $\mathrm{pH} 4$, los copos de nieve comienzan a redisolverse y el sistema adquiere una textura homogénea de color lechoso. A partir de este valor es difícil identificar individualmente cada unidad de fase sólida, como si fue posible hacerlo en la primera región.

En la región $\mathrm{BC}$ se reduce bastante la eficacia del proceso de conformación de complejos de aluminio y por lo tanto la formación de núcleos de fase sólida (el consumo de $\mathrm{OH}^{-}$por parte del sistema disminuye bastante). En el otro paso, región $\mathrm{CD}$, se mantiene la textura homogénea y el color lechoso del sistema.

La Fig. 2 muestra como se modifican las curvas de valoración potenciométrica al variar la concentración ini-

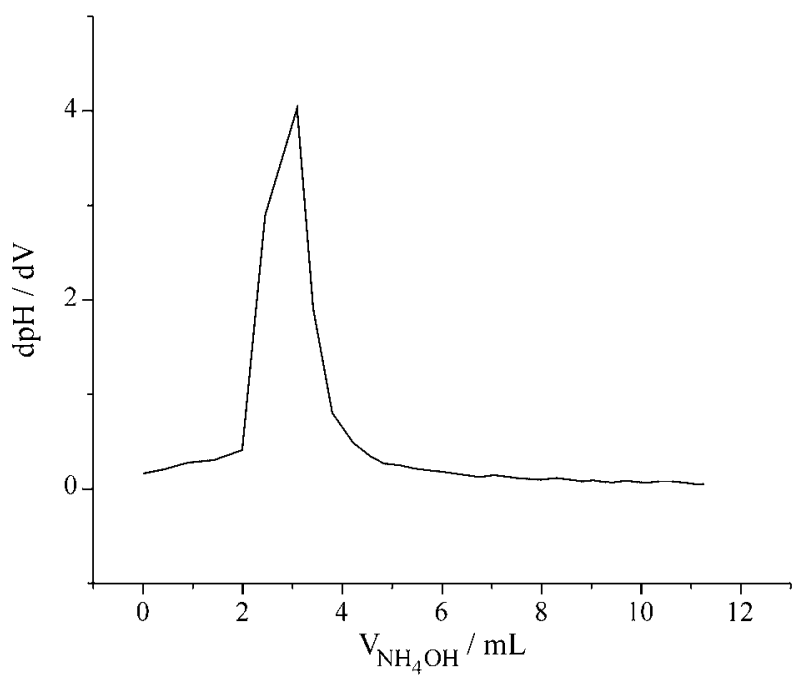

Figure 1b. Primera derivada de la cura potenciométrica para una disolución 0.02 molar de $\mathrm{Al}_{2}\left(\mathrm{SO}_{4}\right)_{3}$. 


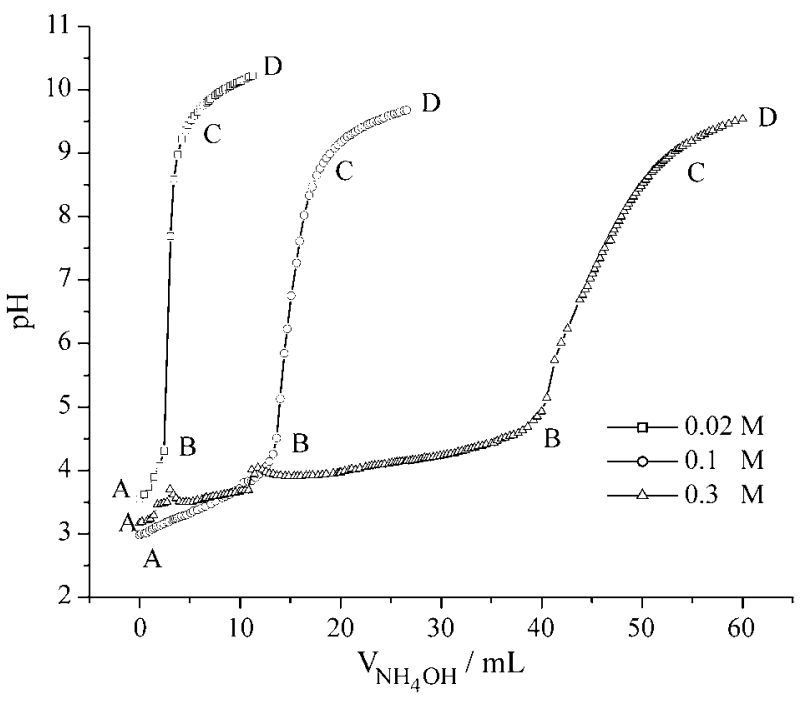

Figure 2. Curvas potenciométricas para diferentes concentraciones de $\mathrm{Al}_{2}\left(\mathrm{SO}_{4}\right)_{3}$.

cial de $\mathrm{Al}_{2}\left(\mathrm{SO}_{4}\right)_{3}$. En todas ellas son evidentes las tres regiones de la Fig. 1a y que fueron analizadas previamente. La extensión de la región A'B' se incrementa a medida que se aumenta la concentración inicial de precursor. Dado que la nucleación es el proceso más importante en esta región, es de esperar que la cantidad de núcleos que se formen sea mayor para el sistema que tenga una mayor concentración de $\mathrm{Al}_{2}\left(\mathrm{SO}_{4}\right)_{3}$. El valor de $\mathrm{pH}$ correspondiente al punto $\mathrm{A}$ es aproximadamente 3.2 independiente de la concentración.

$\mathrm{Al}$ incrementar la concentración inicial del precursor de aluminio, con pocos mililitros de $\mathrm{NH}_{4} \mathrm{OH}$ adicionados al sistema se forman partículas irregulares pequeñas que se observan a simple vista. Estas se van tornando aproximadamente esféricas a medida que se avanza en el proceso de adición de la base. El tamaño de las partículas iniciales se incrementa al aumentar la concentración de $\mathrm{Al}_{2}\left(\mathrm{SO}_{4}\right)_{3}$. No hay evidencia visual de aglomeración. El tamaño de las partículas va disminuyendo a medida que se adiciona el $\mathrm{NH}_{4} \mathrm{OH}$ y a un valor de $\mathrm{pH}$ aproximadamente igual a 3.8 , cerca al punto $\mathrm{B}$, el sistema adquiere un color blanco dificultándose la observación de las unidades sólidas.

El salto de $\mathrm{pH}$ en la región $\mathrm{BC}$ es de aproximadamente 5.0 unidades independiente de la concentración inicial de $\mathrm{Al}_{2}\left(\mathrm{SO}_{4}\right)_{3}$. Para concentraciones altas es más evidente, visualmente, la disminución del tamaño de las partículas y el incremento en número de las mismas. Por lo mencionado, el proceso de redisolución de la fase sólida debe ser muy importante en esta región. A un valor de $\mathrm{pH}$ aproximadamente igual a 8.8 , cerca al punto $\mathrm{C}$, el sistema se torna completamente lechoso.

\subsection{Valoración conductimétrica}

En la Fig. 1a se observa la gráfica que representa la variación de la conductividad específica en función del volumen de $\mathrm{NH}_{4} \mathrm{OH}$ adicionado al sistema. En ella se distinguen claramente dos regiones cercanamente lineales: una región, $\mathrm{AB}$, relacionada con las regiones $\mathrm{AB}$ y $\mathrm{BC}$ de la curva de valoración potenciométrica, y la región $\mathrm{BC}$. $\mathrm{La}$ aparente linealidad de estas regiones se debe a la alta concentración del $\mathrm{NH}_{4} \mathrm{OH}, 15 \mathrm{M}$, utilizado como precipitante.

La región A'B' es cercanamente lineal y dentro de ella se presentan leves variaciones de pendiente a un valor aproximado de $0.9 \mathrm{~mL}$ y a $2.5 \mathrm{~mL}$. Esta región debe corresponder a la valoración de grupos funcionales de naturaleza ácido débil presentes en el sistema. En realidad, grupos superficiales ácido débil se producen por la hidrólisis de los grupos sulfato superficiales a través de la reacción de Kolthoff ${ }^{26}$ :

$$
\equiv \mathrm{SO}_{4}{ }^{-}+\mathrm{H}_{2} \mathrm{O} \rightarrow \equiv \mathrm{HSO}_{4}{ }^{-}+\mathrm{OH}^{-}
$$

donde $\equiv$ representa grupos superficiales y la oxidación de los iones hidroxilo producidos se logra aún en sistemas con poco oxígeno a través de la reacción

$$
2 \mathrm{OH}^{-} \rightarrow \mathrm{H}_{2} \mathrm{O}+1 / 2 \mathrm{O}_{2}
$$

La presencia de especies ácido débil, $\equiv \mathrm{HSO}_{4}{ }^{-}$en la superficie de los coloides, y $\mathrm{HSO}_{4}{ }^{-}$en el seno de la disolución, se puede justificar con base en las curvas de valoración potenciométrica, Fig. 2. El salto de $\mathrm{pH}$ en la zona $\mathrm{BC}$ no es muy grande, $\Delta \mathrm{pH}$ tiene para este caso un valor aproximado de 5, mientras que si existieran grupos superficiales de ácido fuerte en el sistema (grupos $\mathrm{SO}_{4}{ }^{-}$) se debería tener un salto de aproximadamente 7 unidades de $\mathrm{pH}^{27}$. Como la fase sólida se va formando progresivamente, el número de iones en el sistema se incrementa por lo que la conductividad en la región $\mathrm{AB}$ aumenta.

El comienzo de la región B'C' es curvada lo que indica que hay una contribución a la conductividad de una cantidad mayor de iones $\mathrm{NH}_{4}{ }^{-}$y $\mathrm{OH}^{-}$y la disociación del agua ${ }^{28}$. La región B' C' representa la valoración del exceso de base adicionada al sistema después del punto de equivalencia. La base débilmente ionizada que se adiciona a la disolución de la sal que se ha formado, ocasiona que la conductividad alcance un nivel "off" o de muy poca variación.

La Fig. 3 muestra como varia la conductividad específica del sistema al incrementar la concentración inicial del $\mathrm{Al}_{2}\left(\mathrm{SO}_{4}\right)_{3}$ en la disolución. Las características indicadas para la curva de valoración conductimétrica de la Fig. 1a se mantienen. Al incrementar la concentración es más evidente el cambio de pendiente en el interior de la región A'B': por ejemplo en el sistema con una concen- 


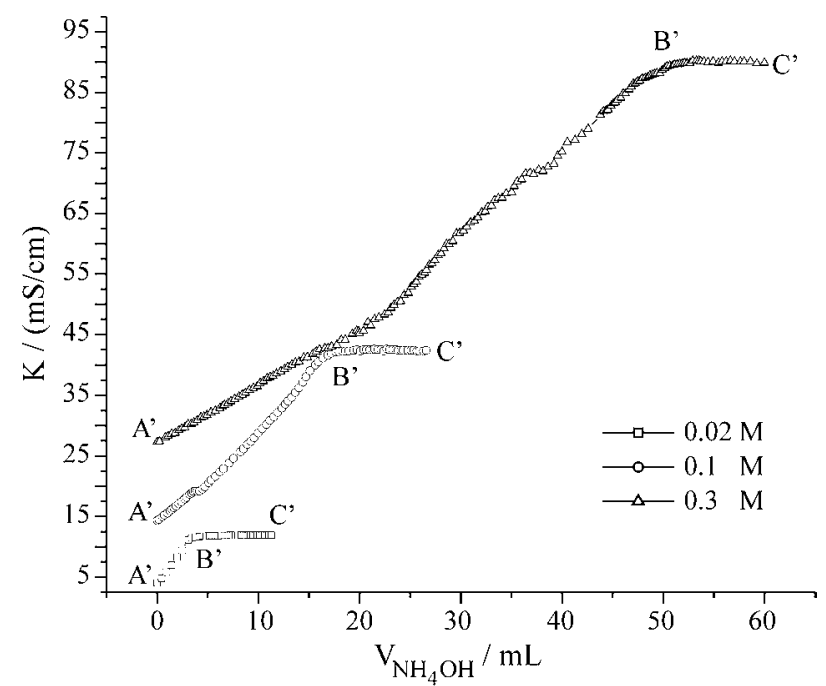

Figure 3. Curvas conductimétricas para diferentes concentraciones de $\mathrm{Al}_{2}\left(\mathrm{SO}_{4}\right) 3$.

tración $0.3 \mathrm{M}$ aproximadamente entre $23 \mathrm{~mL}$ y $39 \mathrm{~mL}$. Además, los tramos internos de la región $\mathrm{AB}$ no son completamente lineales lo que indica la presencia de grupos poliméricos ionizables.

\subsection{Hidrólisis y precipitación del aluminio}

En medios ácidos predomina el $\mathrm{Al}^{3+}$ que coexiste con los cationes básicos $\mathrm{AlOH}^{2+}$ y $\mathrm{Al}(\mathrm{OH})_{2}{ }^{+29,30,31,32}$. Estas especies, y el acusado carácter ácido del $\mathrm{Al}^{3+}$, ocasionan la precipitación de sales básicas de aluminio antes que hidróxido de aluminio. Además, bajo las condiciones a las que se realizaron los ensayos, los sulfatos básicos de aluminio son menos solubles que el hidróxido de aluminio o el sulfato de aluminio sólo ${ }^{23}$. Estos complejos mononucleares deben existir en mayor cantidad en el sistema que contiene una concentración de $0.02 \mathrm{M}$ de $\mathrm{Al}_{2}\left(\mathrm{SO}_{4}\right)_{3}{ }^{31,32}$. $\mathrm{El} \mathrm{Al}(\mathrm{OH})_{2}{ }^{+}$aparece por encima de un valor de $\mathrm{pH} 3$ y se forma rápida y reversiblemente ${ }^{30}$. Considerando los resultados de Kubota ${ }^{31}$ esta especie se obtiene a partir de la siguiente reacción:

$$
\mathrm{Al}^{3+}+\mathrm{H}_{2} \mathrm{O} \leftrightarrow \mathrm{AlOH}^{2+}+\mathrm{H}^{+}
$$

La otra especie básica se formaría a través de la siguiente reacción ${ }^{30,31}$

$$
\mathrm{Al}^{3+}+2 \mathrm{H}_{2} \mathrm{O} \leftrightarrow \mathrm{Al}(\mathrm{OH})_{2}^{+}+2 \mathrm{H}^{+}
$$

o por reacción de las especies $\mathrm{Al}(\mathrm{OH})_{2}{ }^{+}$según:

$$
\mathrm{Al}(\mathrm{OH})_{2}{ }^{+}+\mathrm{H}_{2} \mathrm{O} \leftrightarrow \mathrm{Al}(\mathrm{OH})_{2}{ }^{+}+\mathrm{H}^{+}
$$

Los protones $\mathrm{H}^{+}$que se generan durante la formación de estas especies mononucleares neutralizarían los $\mathrm{OH}^{-}$ producidos por la disociación del $\mathrm{NH}_{4} \mathrm{OH}$ que se adiciona a la disolución. Esto justifica, en parte, el alto consumo de iones hidróxilo en la región $\mathrm{AB}$.
$\mathrm{Al}$ incrementar la concentración inicial de $\mathrm{Al}_{2}\left(\mathrm{SO}_{4}\right)_{3}$, la formación de complejos hidroxopolinucleares es el proceso más importante ${ }^{30,32,33}$. Desafortunadamente no existe un consenso general sobre la identidad exacta y el número de tales especies poliméricas en disolución. Algunos autores consideran que en el sistema existe una combinación de monómeros, dímeros y trímeros con especies poliméricas del tipo $\mathrm{Al}_{13}(\mathrm{OH})_{32}{ }^{7+} \mathrm{o} \mathrm{Al}_{14}(\mathrm{OH})_{34}{ }^{8+}{ }^{32,33}$. En el trabajo de Matijevic y colaboradores ${ }^{32,33}$ se indica que el primer paso en la hidrólisis del aluminio estaría representado por la siguiente reacción:

$$
8 \mathrm{Al}^{3+}+20 \mathrm{H}_{2} \mathrm{O} \leftrightarrow \mathrm{Al}_{8}(\mathrm{OH})_{20}^{4+}+2 \mathrm{OH}^{+}
$$

y que el paso siguiente en la hidrólisis sería ${ }^{32,33}$ :

$$
\mathrm{Al}_{8}(\mathrm{OH})_{20}{ }^{4+}+2 \mathrm{H}^{+} \leftrightarrow \mathrm{Al}(\mathrm{OH})_{22}{ }^{2+}
$$

que con la adición posterior de iones de $\mathrm{OH}^{-}$, e incorporación en los complejos, puede llevar a la formación de la especie neutra $\mathrm{Al}_{8}(\mathrm{OH})_{24}$.

Otras especies polinucleares que también se referencian son el $\mathrm{Al}_{2}(\mathrm{OH})_{2}{ }^{4+}, \mathrm{Al}_{13} \mathrm{O}_{4}(\mathrm{OH})_{24}{ }^{7+}$ y el $\mathrm{Al}_{3}(\mathrm{OH})_{4}{ }^{5+29,30}$, que predominarían entre valores de $\mathrm{pH} 3.5$ y 4.5 . La primera especie polinuclear permite garantizar la formación de un sulfato básico de aluminio, $\left[\mathrm{Al}_{2}(\mathrm{OH})_{2}\left(\mathrm{H}_{2} \mathrm{O}\right)_{8}\right]\left(\mathrm{SO}_{4}\right)_{2}$ en el sistema ${ }^{30}$. Esta sal básica de aluminio se podría conformar en la región $\mathrm{AB}$ de las curvas de valoración y permitiría justificar el gran consumo de $\mathrm{OH}^{-}$. Por otro lado la especie polinuclear $\mathrm{Al}_{13} \mathrm{O}_{4}(\mathrm{OH})_{24}{ }^{7+}$ permite la formación del ión $\mathrm{AlO}_{13}{ }^{7+15}$ que conformaría otros sulfatos básicos de aluminio ${ }^{30}$.

A altos valores de $\mathrm{pH}$ la especie predominante de aluminio en el sistema es el $\mathrm{AlO}_{2}{ }^{-29,30}$. Por otro lado, ya que el $\mathrm{Al}^{3+}$ no forma complejos aminados, el $\mathrm{NH}_{3}$ se comporta únicamente como reactivo básico ${ }^{29}$. Un ácido débil como el catión amonio, y considerando que la disolución reguladora $\mathrm{NH}_{4}{ }^{+} / \mathrm{NH}_{3}$ tiene un $\mathrm{pH}$ de aproximadamente 9, puede ocasionar la precipitación de hidróxido de aluminio a través de la siguiente reacción ${ }^{29}$ :

$$
\mathrm{AlO}_{2}^{-}+\mathrm{NH}_{4}^{+}+\mathrm{H}_{2} \mathrm{O} \rightarrow \mathrm{Al}(\mathrm{OH})_{3} \downarrow+\mathrm{NH}_{3}
$$

Considerando lo mencionado anteriormente se puede concluir que, durante la adición del $\mathrm{NH}_{4} \mathrm{OH}$ a la disolución de $\mathrm{Al}_{2}\left(\mathrm{SO}_{4}\right)_{3}$, a medida que aumenta el $\mathrm{pH}$, los iones $\mathrm{Al}^{3+}$ (acuo) irán formando iones polinucleares complejos a través del proceso de olación $\left(\mathrm{M}-\mathrm{OH}+\mathrm{M}-\mathrm{OH}_{2} \rightarrow \mathrm{M}-\mathrm{OH}-\right.$ $\left.\mathrm{M}+\mathrm{H}_{2} \mathrm{O}\right)$ y oxolación $\left(\mathrm{M}-\mathrm{OH}+\mathrm{M}-\mathrm{OH} \rightarrow \mathrm{M}-\mathrm{O}-\mathrm{M}+\mathrm{H}_{2} \mathrm{O}\right)$ y que finalmente se conformarán, por interacción entre las especies de iones, complejos polinucleares de sulfatos básicos de aluminio. Estos complejos polinucleares actuarían como precursores en la formación de los embriones ${ }^{20,24}$. Ellos se irán formando a medida que se adiciona el $\mathrm{NH}_{4} \mathrm{OH}$ a la solución, principalmente en la zona $\mathrm{AB}$ de las curvas 
de valoración potenciómetrica (Figs. 1 y 2). Por último, las especies conformarían los núcleos de fase sólida por la condensación de los compuestos que continuamente se van formando en la suspensión coloidal.

Al ir aumentando la concentración de hidróxido de amonio en el sistema se redisuelve la sal básica y se forma un aluminato. Ya que el ión sulfato es un ión "penetrante" en las especies básicas de aluminio ${ }^{21}$, éste se intercambiará fácilmente con los iones hidroxilos favoreciendo la formación del aluminato que posteriormente se transformará en un oxihidróxido de aluminio por la acción adicional del catión amonio (ácido débil) a altos valores de $\mathrm{pH}$.

Con relación a los grupos superficiales que existen sobre los coloides, las reacciones mas importantes que pueden ocurrir $\operatorname{son}^{34}$ :

$$
\equiv \mathrm{Al}(\mathrm{OH})+\mathrm{H}^{+}+\mathrm{SO}_{4}{ }^{2-} \leftrightarrow \equiv \mathrm{AlSO}_{4}{ }^{-}+\mathrm{H}_{2} \mathrm{O}
$$

en condición ácida favoreciendo la formación de grupos superficiales ácido fuerte $\mathrm{y}$

$$
\equiv \mathrm{AlOH}+\mathrm{SO}_{4}{ }^{2-} \leftrightarrow \equiv \mathrm{AlOHSO}_{4}{ }^{2-}
$$

garantizando la formación de ácidos débiles. $\mathrm{El} \mathrm{SO}_{4}{ }^{2-}$ está fuertemente ligado a la superficie de la alúmina bajo condiciones ácidas o ligeramente ácidas.

\subsection{Caracterización de la fase líquida}

Para determinar especies químicas o grupos funcionales presentes en el sistema $\mathrm{Al}_{2}\left(\mathrm{SO}_{4}\right)_{3}-\mathrm{NH}_{4} \mathrm{OH}-\mathrm{H}_{2} \mathrm{O}$ se caracterizó la fase líquida, obtenida al filtrar la suspensión coloidal a diferentes valores de $\mathrm{pH}$, utilizando espectroscopia UV-visible e infrarroja. La Tabla 1 resume los datos de UV-visible obtenidos, indicándose la ubicación de las bandas y los valores de absorbancia para las diferentes muestras analizadas del sistema $0.1 \mathrm{M}$ de $\mathrm{Al}_{2}\left(\mathrm{SO}_{4}\right)_{3}$. A pH 4.11 las principales especies son las polinucleares, $\mathrm{Al}_{2}(\mathrm{OH})_{2}{ }^{4+}$ y $\mathrm{Al}_{3}(\mathrm{OH})_{4}{ }^{5+}$, y los polímeros de aluminio, $\mathrm{Al}_{13}(\mathrm{OH})_{32}{ }^{7+}, \mathrm{Al}_{13} \mathrm{O}_{4}(\mathrm{OH})_{24}{ }^{7+}$, ó $\mathrm{Al}_{14}(\mathrm{OH})_{34}{ }^{8+}$, que participan en la formación de la sal básica de aluminio. El espectro presenta una banda muy ancha para longitudes de onda por encima de los $280 \mathrm{~nm}$ y el máximo de absorción se presenta a $356 \mathrm{~nm}(0,224$ unidades de absorbancia).

La muestra tomada en el punto de equivalencia, $\mathrm{pH} 7.7$, presenta varias absorciones entre 200 y $300 \mathrm{~nm}$ destacán-

Tabla 1. Resultados de espectroscopia UV-visible de la fase líquida del sistema $\mathrm{Al}_{2}\left(\mathrm{SO}_{4}\right)_{3}-\mathrm{NH}_{4} \mathrm{OH}-\mathrm{H}_{2} \mathrm{O}$ a diferentes valores de $\mathrm{pH}$.

\begin{tabular}{lcc}
\hline $\mathrm{pH}$ & $\lambda(\mathrm{nm})$ & Absorbancia \\
\hline 4.11 & 356 & 0.224 \\
7.7 & $239 ; 308$ & $0.102 ; 0.200$ \\
10.52 & 308 & 0.141 \\
\hline
\end{tabular}

dose las de $239 \mathrm{~nm}$ (0.102 unidades de absorbancia), $266 \mathrm{~nm}$ (0.104 unidades de absorbancia), $275 \mathrm{~nm}$ (0.107 unidades de absorbancia), y otra a $308 \mathrm{~nm}$ (0.2 unidades de absorbancia). Las tres primeras absorciones son muy débiles y su intensidad está un poco por encima del nivel de ruido del espectro. A un $\mathrm{pH}$ de 10.52 la banda a $308 \mathrm{~nm}$ se mantiene, disminuyendo su intensidad (0.141 unidades de absorbancia), y no se aprecia ninguna absorción por encima de $310 \mathrm{~nm}$. En la Fig. 4 se muestran espectros de UV-visible característicos de las muestras analizadas.

Los cambios que presentan los resultados de UV-visible se pueden justificar considerando las variaciones en las características de absorción de las especies químicas presentes o que las especies que existen en el sistema modifican su estructura continuamente durante los procesos de nucleación, precipitación y redisolución. Indudablemente los espectros (b) y (c) de la Fig. 4 deben corresponder a especies solubles presentes en la formación de la sal básica de aluminio y el espectro (c) debe contener, además, información sobre las características de absorción de las especies solubles existentes en el sistema durante la formación del aluminato.

En la Fig. 5 se indican los espectros IR de las muestras líquidas obtenidas al filtrar el sistema $\mathrm{Al}_{2}\left(\mathrm{SO}_{4}\right)_{3}-\mathrm{NH}_{4} \mathrm{OH}$ - $\mathrm{H}_{2} \mathrm{O}$, a diferentes valores de $\mathrm{pH}$ y para una concentración $0.1 \mathrm{M}$ de sulfato de aluminio. En estos espectros son evidentes las bandas del agua, $3265 \mathrm{~cm}^{-1}$ y $1635 \mathrm{~cm}^{-1}$, y la $v_{4}$ del $\mathrm{NH}_{4}^{+}$, a $1456 \mathrm{~cm}^{-1}$, para valores altos de $\mathrm{pH}$ y que corresponde al $\left(\mathrm{NH}_{4}\right)_{2} \mathrm{SO}_{4}$ que se forma en el sistema. $\mathrm{La}$ banda a $1100 \mathrm{~cm}^{-1}$ se puede asignar al $\nu_{3}$ del $\mathrm{SO}_{4}{ }^{2-}$ libre y la de $613 \mathrm{~cm}^{-1}$ al $v_{4}$ de este mismo ión ${ }^{35}$. La banda ubicada a $781 \mathrm{~cm}^{-1}$ corresponde al modo vibracional de tensión del enlace Al- $\mathrm{O}^{36}$ y la ubicada a $810 \mathrm{~cm}^{-1}$ se asigna a la vibracional de tensión del enlace $\mathrm{Al}-\mathrm{O}-\mathrm{Al}^{36}$. Estos resultados indican que en el líquido existen especies solubles de aluminio, sulfato y $\left(\mathrm{NH}_{4}\right)_{2} \mathrm{SO}_{4}$, principalmente.

\subsection{Caracterización de la fase sólida}

Los espectros FTIR de muestras sólidas obtenidas al filtrar el sistema $\mathrm{Al}_{2}\left(\mathrm{SO}_{4}\right)_{3}-\mathrm{NH}_{4} \mathrm{OH}-\mathrm{H}_{2} \mathrm{O}$, a diferentes

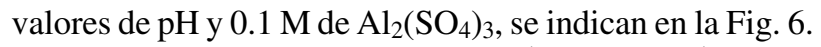
La banda más intensa entre $3053 \mathrm{~cm}^{-1}$ a $3564 \mathrm{~cm}^{-1}$ corresponde a las vibraciones de tensión del $\mathrm{O}-\mathrm{H}$ del agua (modo simétrico $v_{1} \mathrm{y}$ antisimétrico $v_{3}$ ) mientras que la banda a $1647 \mathrm{~cm}^{-1}$ se asocia al modo de flexión del $\mathrm{H}-\mathrm{O}-\mathrm{H}^{35}$. La ubicación de las bandas que se asocian al $\mathrm{SO}_{4}{ }^{2-}$, y la presencia o no de las mismas en el espectro, permite obtener información sobre la naturaleza del complejo del cual forma parte el ión sulfato. Mientras el $\mathrm{SO}_{4}{ }^{2-}$ libre corresponde al grupo puntual $\mathrm{T}_{\mathrm{d}}$ de alta simetría, y sólo dos de los cuatro modos fundamentales son activos en el infrarrojo 

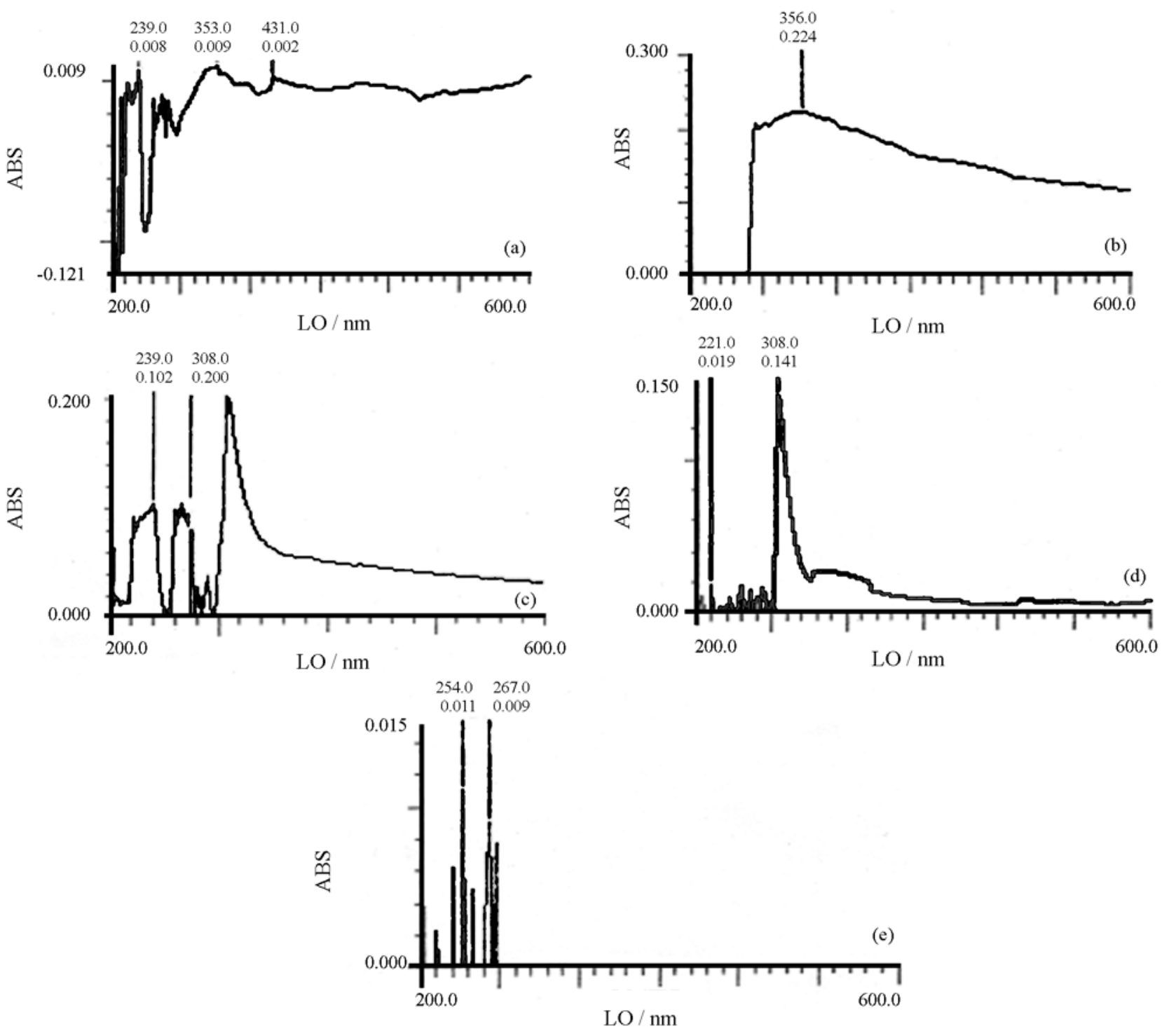

Figure 4. Espectros de UV-visible característicos de una disolución de $\mathrm{Al}_{2}\left(\mathrm{SO}_{4}\right)_{3} 0.1$ molar, correspondientes a muestras líquidas obtenidas del filtrado del sistema $\mathrm{Al}_{2}\left(\mathrm{SO}_{4}\right)_{3}-\mathrm{NH}_{4} \mathrm{OH}-\mathrm{H}_{2} \mathrm{O}$ a diferentes valores de $\mathrm{pH}$ : (a) $\mathrm{pH} 3$, (b) $\mathrm{pH} 7.7$ y (c) $\mathrm{pH} 10.52$.

( $v_{3}$ y $\left.v_{4}\right)$, cuando entra a formar parte de algún complejo la simetría disminuye, las vibraciones se degeneran y se separan y los modos activos en Raman aparecen en el espectro de infrarojo ${ }^{37}$. La disminución de la simetría, causada por la coordinación del ión sulfato, es diferente para los complejos unidentados y para los complejos bidentados, ésto permite la distinción entre uno y otro.

Para la muestra sólida obtenida a pH 3, Fig. 6a, el complejo de sulfato presenta las bandas $v_{3}, 1123 \mathrm{~cm}^{-1} \mathrm{y}$ $1015 \mathrm{~cm}^{-1}, v_{4}, 614 \mathrm{~cm}^{-1}$ y $664 \mathrm{~cm}^{-1}$, como un pequeño hombro la $v_{1}, 973 \mathrm{~cm}^{-1}$ y $v_{2}, 460 \mathrm{~cm}^{-1}$, muy pequeña. De acuerdo a la bibliografía ${ }^{37}$ esta muestra contendría complejos bidentados de $\mathrm{SO}_{4}{ }^{2-}$. En los espectros de FTIR de las muestras sólidas a pH 5 y 10, Figs. 6 b y 6c, es evidente la desaparición de la banda $v_{2}, 460 \mathrm{~cm}^{-1}$, cuando aumenta el $\mathrm{pH}$ de la muestra. La presencia de la $v_{1}, 976 \mathrm{~cm}^{-1}$, es más evidente en estas dos muestras así como la presencia de una sola banda $v_{3}, 1115 \mathrm{~cm}^{-1}$, en la muestra $\mathrm{pH} 10$. Estos resultados indican que los complejos de $\mathrm{SO}_{4}{ }^{2-}$ que predominan a altos valores de $\mathrm{pH}$ son los unidentados. Lo anterior está de acuerdo con que el ión $\mathrm{SO}_{4}{ }^{2-}$, en los compuestos básicos de aluminio se puede intercambiar fácilmente ${ }^{20} \mathrm{y}$ como el ión no actúa como puente, entre especies polinucleares hidrolizadas, el enlace con el complejo es débil.

Las bandas $3040 \mathrm{~cm}^{-1}\left(v_{1}\right), 1679 \mathrm{~cm}^{-1}\left(v_{2}\right), 1401 \mathrm{~cm}^{-1}$ $\left(v_{4}\right)$ corresponde a la molécula tetraedral $\mathrm{NH}_{4}{ }^{+36}$. Las bandas del espectro que se encuentran a más alta frecuencia corresponden a iones hidroxilos aislados que se encuentran 




Figure 5a. Espectro IR característico de una disolución de $\mathrm{Al}_{2}\left(\mathrm{SO}_{4}\right)_{3}$ 0.1 molar, correspondientes a la muestra líquida obtenida del filtrado del sistema $\mathrm{Al}_{2}\left(\mathrm{SO}_{4}\right)_{3}-\mathrm{NH}_{4} \mathrm{OH}-\mathrm{H}_{2} \mathrm{O}$ a pH 3 .



Figure 5b. Espectro IR característico de una disolución de $\mathrm{Al}_{2}\left(\mathrm{SO}_{4}\right)_{3}$ 0.1 molar, correspondientes a muestras líquidas obtenidas del filtrado del sistema $\mathrm{Al}_{2}\left(\mathrm{SO}_{4}\right)_{3}-\mathrm{NH}_{4} \mathrm{OH}-\mathrm{H}_{2} \mathrm{O}$ a pH 4.11 .

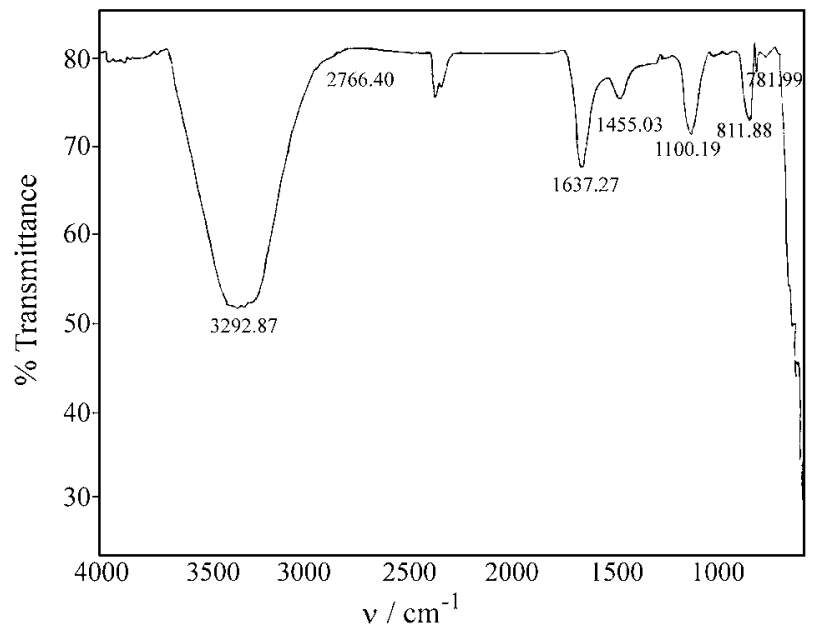

Figure 5c. Espectro IR característico de una disolución de $\mathrm{Al}_{2}\left(\mathrm{SO}_{4}\right)_{3}$ 0.1 molar, correspondientes a muestras líquidas obtenidas del filtrado del sistema $\mathrm{Al}_{2}\left(\mathrm{SO}_{4}\right)_{3}-\mathrm{NH}_{4} \mathrm{OH}-\mathrm{H}_{2} \mathrm{O}$ a pH 8.79 . en la superficie de las partículas: la banda a $3862 \mathrm{~cm}^{-1}$ correspondería a un hidroxilo con cuatro oxígenos primeros vecinos y la banda ubicada a $3742 \mathrm{~cm}^{-1}$ al hidroxilo con dos oxígenos primeros vecinos ${ }^{38}$. Observando cuidadosamente el espectro correspondiente a la muestra sólida obtenida a pH 10, cerca a la banda de $915 \mathrm{~cm}^{-1}$, se encuentra un hombro pequeño a $960 \mathrm{~cm}^{-1}$ y otro a $760 \mathrm{~cm}^{-1}$.
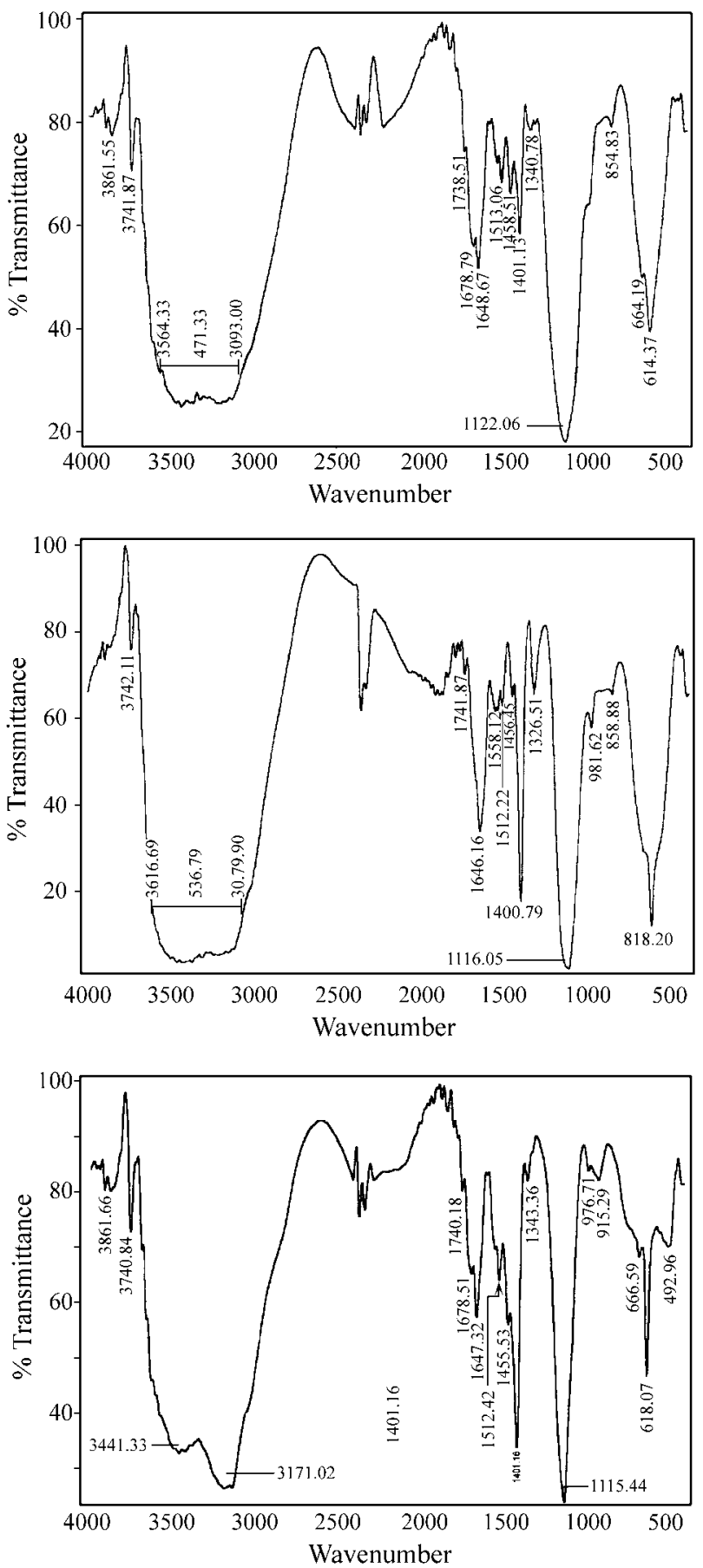

Figure 6. Espectros IR de fases sólidas obtenidas de la disolución 0.1 molar de $\mathrm{Al}_{2}\left(\mathrm{SO}_{4}\right)_{3}$ a diferentes pH: (a) pH 3, (b) $\mathrm{pH} 5$ y (c) $\mathrm{pH} 10$. 


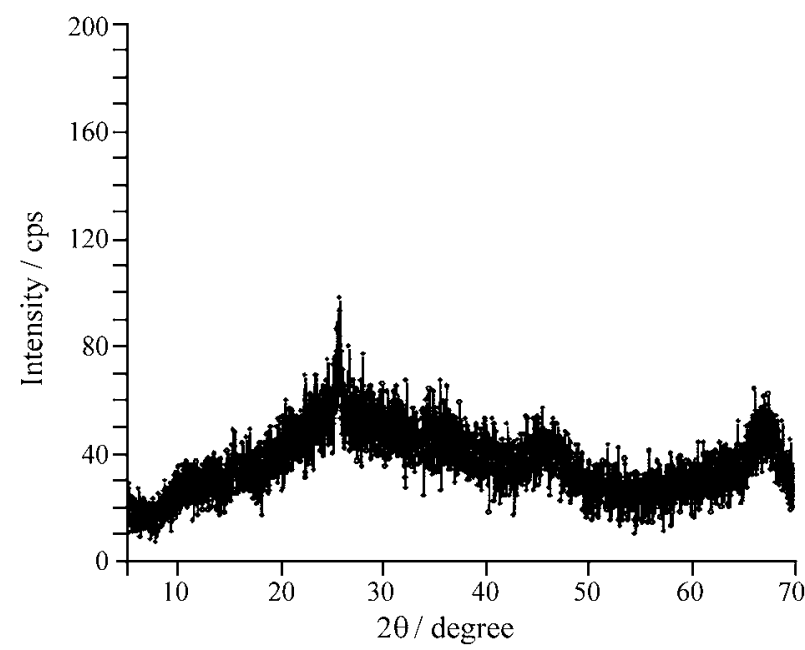

Figure 7. Difractograma de rayos-x de la fase sólida obtenida de una disolución 0.1 molar de $\mathrm{Al}_{2}\left(\mathrm{SO}_{4}\right)_{3}$ un $\mathrm{pH} 10$ después de dejarla envejecer dos días en el líquido madre.

Estas bandas son características de la gibbsita, $\mathrm{Al}(\mathrm{OH})_{3}$, lo que lleva a considerar su presencia en la muestra. Estas bandas, más otra que estaría solapada por la de $1115 \mathrm{~cm}^{-1}$, corresponderían a las cuatro vibraciones de deformación del $\mathrm{OH}$ del $\mathrm{Al}(\mathrm{OH})_{3}{ }^{36}$ : la banda a $960 \mathrm{~cm}^{-1}$ se asigna a la vibración Al-OH-Al y la banda $760 \mathrm{~cm}^{-1}$ a la vibración Al-OH. En esta muestra coexisten compuestos de sulfato básico de aluminio con hidróxido de aluminio, lo que permite la transformación de la especie básica de aluminio en hidróxido de aluminio por la acción del catión amonio a valores altos del $\mathrm{pH}^{29}$.

En la Fig. 7 se ilustra el difractograma de rayos- $\mathrm{x}$ correspondiente a la muestra sólida obtenida de la suspen-

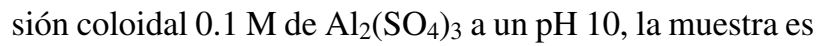
amorfa.

\section{Conclusiones}

Del estudio realizado con valoración potenciométrica y conductimétrica y de la caracterización tanto del líquido de filtrado como de la fase sólida del sistema se puede concluir lo siguiente:

a) Las curvas de valoración potenciométrica del sistema $\mathrm{Al}_{2}\left(\mathrm{SO}_{4}\right)_{3}-\mathrm{NH}_{4} \mathrm{OH}-\mathrm{H}_{2} \mathrm{O}$ presentan tres regiones bien definidas. La región inicial, primer peldaño, presenta un alto consumo de $\mathrm{OH}^{-}$lo que indica la formación de especies mononucleares como el $\mathrm{AlOH}^{2+}$ y el $\mathrm{Al}(\mathrm{OH})_{2}{ }^{+}$, principalmente a bajas concentraciones del precursor de aluminio, $\mathrm{y}$ de especies polinucleares, $\mathrm{Al}_{2}(\mathrm{OH})_{2}{ }^{4+}$ y $\mathrm{Al}_{3}(\mathrm{OH})_{4}{ }^{5+}$, y poliméricas $\mathrm{Al}_{13}(\mathrm{OH})_{32}{ }^{7+}, \mathrm{Al}_{13} \mathrm{O}_{4}(\mathrm{OH})_{24}{ }^{7+} \mathrm{o} \mathrm{Al}_{14}(\mathrm{OH})_{34}{ }^{8+}$, a altas concentraciones.

En esta primera región se formarían sulfatos básicos de aluminio a través de reacciones del siguiente tipo:

$$
\begin{array}{rl}
\mathrm{Al}_{2}\left(\mathrm{SO}_{4}\right)_{3}+\underset{ }{2} & 2 \mathrm{NH}_{4} \mathrm{OH}+8 \mathrm{H}_{2} \mathrm{O} \rightarrow \\
{\left[\mathrm{Al}_{2}(\mathrm{OH})_{2}\left(\mathrm{H}_{2} \mathrm{O}\right)_{8}\right]\left(\mathrm{SO}_{4}\right)_{2}+\left(\mathrm{NH}_{4}\right)_{2} \mathrm{SO}_{4}}
\end{array}
$$

Estos sulfatos básicos actuarían como precursores en la formación de los embriones de la fase sólida y por lo tanto de los núcleos.

En la segunda región se presentaría la transformación del sulfato básico de aluminio en un aluminato y en la última región se debería formar $\mathrm{Al}(\mathrm{OH})_{3}$. Estas transformaciones se ven favorecidas por el carácter de ión penetrante que presenta $\mathrm{el} \mathrm{SO}_{4}{ }^{2-}$ en los aluminatos básicos, lo que hace que sea fácilmente intercambiable con los iones hidroxilos.

b) La curva de valoración conductimétrica posee dos regiones bien definidas. En la primera región existen tramos lineales que presentan variaciones de pendiente. Esta región corresponde a la valoración de especies en el seno de la disolución, $\mathrm{HSO}_{4}{ }^{-}$y los grupos superficiales $\equiv \mathrm{AlO}-$ $\mathrm{HSO}_{4}{ }^{2-}$ de naturaleza ácido débil. A concentraciones de $\mathrm{Al}_{2}\left(\mathrm{SO}_{4}\right)_{3}$ mayores, los tramos no son completamente lineales poniendo en evidencia la presencia de los grupos poliméricos ionizables.

La segunda región corresponde a la valoración de la base débil adicionada a la suspensión del aluminato. En esta región debe ocurrir la precipitación $\operatorname{del} \mathrm{Al}(\mathrm{OH})_{3}$ y a un $\mathrm{pH}$ aproximado de 9.2, la acción reguladora del $\mathrm{NH}_{4}{ }^{+} / \mathrm{NH}_{3}$; comienza a actuar sobre el sistema.

c) La caracterización del líquido de la suspensión $0.1 \mathrm{M}$ de $\mathrm{Al}_{2}\left(\mathrm{SO}_{4}\right)_{3}$ indica que existen en él especies solubles conformadas por complejos de sulfato-aluminio, además de sulfato de amonio, que se forman a medida que se adiciona $\mathrm{NH}_{4} \mathrm{OH}$ al sistema. El estudio de la fase sólida del sistema pone en evidencia la existencia de compuestos de sulfato básico de aluminio bidentados a bajos $\mathrm{pH}$. Estos compuestos modifican su estructura a monodentados a medida que se adiciona el $\mathrm{NH}_{4} \mathrm{OH}$ y se incrementa el $\mathrm{pH}$. Esto se puede justificar considerando que el $\mathrm{SO}_{4}{ }^{2-}$ no está fuertemente ligado al complejo, y que se puede intercambiar fácilmente con iones $\mathrm{OH}$, favoreciéndose la formación de oxihidróxidos de aluminio a altos valores de $\mathrm{pH}$ del sistema. El espectro de FTIR de la muestra tomada a pH 10 indica presencia de gibbsita en la fase sólida del sistema, justificando lo mencionado anteriormente. La naturaleza amorfa de la muestra hace prever una alta reactividad del polvo cerámico obtenido.

\section{Agradecimientos}

A la Fundación para la promoción de la Investigación y la Tecnología, a la Vicerrectoria de Investigaciones de la Universidad del Cauca por la ayuda económica para realizar este trabajo y a los doctores O.L. Hoyos, por la revisión y comentarios del manuscrito, y N. Rojas por los espectros de UV-visible. 


\section{Bibliografía}

1. Alumina Chemicals: Science and Technology Handbook, Hart, L.D. (ed), The American Ceramic Society, Inc., 1990.

2. British Patent 10,093,1887.

3. Ganguli, D.; Chatterjee, M. Ceramic Powder preparation: A Handbook, Klwver Academic Publishers, 1997.

4. Willard, H.; Tang, N.K. J. Am. Chem. Soc., v. 59, p. 1190-1196, 1937.

5. Nagai, H.; Hozazono, S.; Kato, A. Br. Ceram. Trans. J., v. 90, p. 44-48, 1991.

6. Sarikaya, Y.; Akin, M. Ceram. Int., v. 14, p. 239-244, 1988.

7. Ray, J.; Chatterjee, M.; Ganguli, D. J. Mater Sci. Lett., v. 12, p. 1755-1757, 1993.

8. Wang, L.; Lloyd, I.K. J. Am. Ceram. Soc., v. 74, p. 2934-2936, 1970.

9. Blendell, J.E.; Bowen, H.K.; Coble, R.L. Am. Ceram.Soc. Bull, v. 63, n. 6, p. 797-802, 1984.

10. Sacks, M.; Tseng, T.Y.; Lee, S.Y. Am. Ceram. Soc. Bull., v. 63, p. 301-310, 1981.

11. Nagai, H.; Oshima, Y.; Hirano, K.; Kato, A. Brit. Ceram. Trans, v. 92, p. 114-119, 1993.

12. Matijevic, E. Chem. Mater., v. 5, p. 412-426, 1993.

13. Surface and Colloid Chemistry in advanced ceramics Processing, Pugh, R.J.; Bergstrom, L. (ed), Surfactant Science Series v. 51 Marcel Dekker, Inc., 1994.

14. Otterstedt, J.; Brandreth, D. Small particles Technology, PlenuPress 1998.

15. Johansson, G. Acta Chem. Scand., v. 14, n. 3, p. 771-773, 1960.

16. Bottero, J.Y.; Fiessinger, F. Nordic Pulp and Paper Res.J., n. 2, p. 81, 1989.

17. Bassett, H.; Goodwin, T.H. J. chem.. Soc., p. 2239 , 1949.

18. Gordon, L.; Salustsky, M.L.; Willard, H.N. precipitation from homogeneous solution, Wiley, New York, 1959.

19. Kato, E.; Daimon, K.; Nanbu, M. J. Am. Ceram. Soc., v. 64, n. 8, 436-443, 1981.
20. Brace, R.; Matijevic, E. J. Inorg. Nucl. Chem., v. 35, p. 3691-3705, 1973.

21. Matijevic, E.; Stryker, L.J. J. Colloid Interface Sci., v. 22, p. 68, 1966.

22. Demchak, R.; Matijevic, E. J. Colloid Interface Sci., v. 31, p. 257, 1969.

23. Singh, S.S. Can. J. Soil Sci., v. 49, p. 383, 1969.

24. Tezak, B. Disc. Faraday Soc., v. 42, p. 175-186, 1996.

25. Arai, Y. Chemistry of Powder Production, chapman \& Hall, 1996.

26. Yates, D.E.; Ottewill, R.H.; Goodwin, J.W. J. Colloid Interface. Sci., v. 62, n. 2, p. 356-358, 1997.

27. Stone-Masui; Watillon, A. J. Colloid Interface Sci., v. 52, n. 3, p. 479-503, 1975.

28. Crow, D.R. Principles and Applications of Electrochemistry, Backie Academic \& Professional Fourth edition, 1994.

29. Burriel, F.; Conde, F.; Arribas, S.; Hernández, J. Química Analítica Cualitativa,. Editorial Paraninfo S.A., 1994.

30. Baes, C.F.; Mesmer, R.E. The Hidrolysis of cations, Wiley-Interscience Publication, John Wiley \& Sons. Inc., 1976.

31. Kubota, H. Properties an Volumetric Determination of Aluminum Ion, Dissertation, Univ. of Wisconsin, 1956; Diss. Abstr., v. 16, p. 864, 1956.

32. Matijevic, E.; Mathai, K.G.; Ottewill, R.H.; Kerker, M. J. Phys. Chem., v. 65, p. 826-830, 1961.

33. Vermeulen, A.C.; Geus, J.W.; Stol, R.J.; de Bruyn, P.L. J. Colloid Interface Sci., v. 51, n. 3, p. 449-458, 1975.

34. Stumm W. Chemistry of de solid-water interface, Wiley-Interscience publication, John Wiley \& Sons. Inc., 1992.

35. Nakamoto K. Infrared spectra of inorganic and coordination compounds, John Wiley \& Sons. Inc., 1962.

36. Farmer Y.C. The infrared spectra of minerals, Minerological Society, 1974.

37. Nakamoto K., Infrared and Raman spectra of inorganic and coordination compounds, part A,B, $5^{\text {th }}$ edition, John Wiley \& Sons. Inc., 1997.

38. Brace, R.; Matijevic, E. J. Inorg. Nucl. Chem., v. 35, p. 3691-3705, 1973. 